\title{
Chapter 11 \\ Studying Vulnerable Populations in the Context of Enhanced Vulnerability
}

\author{
Ruth Macklin
}

Brief portions of this article are excerpted from my previously published works: Macklin, Ruth. Double standards in medical research in developing countries. 2004. Cambridge, U.K.: Cambridge University Press; Macklin, Ruth. Ethics and clinical research. 2010. Barcelona, Spain: Fundació Víctor Grífols $i$ Lucas; Macklin, Ruth and Ethan Cowan. 2009. Conducting research in disease outbreaks, PLoS Neglected Tropical Diseases 3:1-3.

\subsection{Critiquing the Need for Research}

Arguments critical of research conducted in disasters focus on one or more of the following points: 1) victims in the midst of a disaster are rendered too vulnerable by the situation to permit their inclusion in research; 2) natural disasters or disease outbreaks in developing countries or poor areas in industrialised countries render the inhabitants even more vulnerable, since what they need is aid, not research; 3) people who are recruited by health workers during a disaster may confuse research with treatment and fall prey to the therapeutic misconception; 4) people caught in a disaster are too emotionally unstable to provide valid informed consent to be a research subject; 5) even following a disaster, those caught in its wake may be traumatised by interviews or physical examinations that cause them to recall terrible circumstances; 6) conducting research in a disaster may impede efforts to mitigate harm and can intrude into rescue operations; and 7) when disaster strikes there is insufficient time to prepare a proper research protocol and have it reviewed by a research ethics committee, so potential subjects may lack adequate protection of their rights and welfare. Responses to these criticisms require a deeper inquiry into the concept of vulnerability, a look at what empirical evidence shows about the mental and emotional state of individuals caught up in disasters, and careful planning to avoid the problems noted in the above criticisms.

\footnotetext{
R. Macklin $(\bowtie)$

Albert Einstein College of Medicine, Bronx, New York, USA

e-mail: ruth.macklin@einstein.yu.edu 


\subsection{The Need for Research in Disasters}

It would take an encyclopaedic volume to describe all the circumstances in various types of disasters where research is needed that can inform better actions in the future. The authors of an article discussing the need for research during or after a terrorist attack provide the following reasons: 'Research that focuses on the effects of terrorism can provide important information that may improve long-term survival, help prepare for subsequent incidents, assess the physical and emotional needs of a population, have an impact on mental health management of victims and other disaster-affected persons, and increase understanding of the human experience' (Fleischman and Wood 2002, p. 315). Three additional illustrations provide more specific details.

The first is a rather technical example regarding exposure to radiation in accidents. The second is a somewhat neglected area: sex and gender differences in vulnerability to disasters and their impact. The third is how best to manage compound fractures and crush injuries in disasters such as earthquakes, which cause many limb fractures.

The authors of a scientific article on accidental radiation exposure begin with the sobering observation that in today's world, there is an enhanced likelihood of accidental radiation exposure to occupational workers, patients and the public (Pandey et al. 2010). Citing the increasing use of nuclear technology in power production, medical and industrial applications, and the possibility of nuclear terrorism or war, the authors describe current knowledge and necessary additional research to improve the treatment and management of victims of radiation accidents. Among other areas where research during radiation accidents can contribute to existing scientific understanding, the article notes that 'there is a need to fill the gaps in knowledge of radiation action in different dose ranges and post-irradiation windows, which would help in improving therapeutic approaches' (Pandey et al. 2010, p. 613). What is striking about the information in the article - at least to a layperson in the field of radiation-are the myriad differences between types of exposure to large doses of radiation. To cite only one example: the radiation exposure from the Chernobyl nuclear accident differed markedly from what the population in Hiroshima and Nagasaki experienced from the atomic bombings. In the atomic bombings, people were exposed to external whole body radiation and minimal exposure from fallout of the radioisotopes. In contrast, in Chernobyl 'millions of people were exposed to a significant level of radioactivity from fallout and external radiation; however, whole body exposure was limited to those working close to the reactor or post-accidental rescue workers' (Pandey et al. 2010, p. 613). Because of these and other differences, the article notes that the expected health consequences to the victims of the Chernobyl accident would be different from those for the atomic bombs survivors. This article was published in August 2010, less than a year before the effects of the earthquake and tsunami devastated the Fukushima Daiichi nuclear plant in Japan. But one can imagine that the circumstances for workers and the public there would differ yet again from those in Chernobyl in 1986 and in Hiroshima and Nagasaki in 1945. After presenting a staggering amount of data on reactions of the human body 
to an array of radioactive substances, Pandey et al. issue a few caveats regarding research during a disaster. One is that casualties of radiation accidents may require the screening of large numbers of individuals - a practical limitation. Other limiting factors are high cost, sophisticated machines, need for trained personnel, and lack of established biomarkers specific to radiation. If we contemplate how clinical studies could practicably have been conducted in the wake of the March 2011 earthquake and tsunami in Japan, the likelihood of such highly sophisticated research appears slim indeed. However, if exposure of large numbers of people occurs near one or more tertiary care hospitals, it may be possible to conduct research while attending scrupulously to the needs of the exposed individuals.

A second illustration is provided by a World Health Organization (WHO) report that noted 'There is a general lack of research on sex and gender differences in vulnerability to and impact of disasters' (World Health Organization 2002). In all likelihood, not much has changed in the intervening decade. Mentioning the limited amount of existing evidence, WHO reported differences between women and men in the negative consequences following a disaster. It is not clear, the report states, whether these are a result of biological differences, socially determined differences, or some combination of the two. Citing an array of statistics and anecdotes, the WHO report paints a picture of greater vulnerability of women than men when disasters strike. The WHO report describes an earthquake in Maharashtra, India, where many more women died than men. Women were inside homes that collapsed, while men were in the open fields during harvest time and boys were away at schools distant from the site of the earthquake. Regarding another well-known phenomenon, the report says that 'Women and girls are more vulnerable to sexual abuse in disaster situations and may be coerced into sex for basic needs such as food, shelter and security. The sex industry often becomes part of the interaction between the refugee or displaced population and the local community' (World Health Organization 2002). Despite these and numerous other known circumstances differentiating the consequences of disasters for women and men, WHO points to the need for additional research at local, national, and international levels. At the local level, research is needed on how gender relations operate in households and communities in a disaster situation and during the relief and recovery phase. Another area that needs to be better researched in the wake of disasters is the special needs of disadvantaged women, such as women with disabilities and women in violent relationships. Even within disaster organisations, studies are needed to determine the effects of gendered organisational culture on staffing, funding, programming, and training. This WHO report is an eye-opening reminder that even in the $21^{\text {st }}$ century, there remains substantial neglect of the type of research that addresses gender equity in situations such as disasters.

A third area where research is needed is how best to manage compound fractures and crush injuries in disasters such as earthquakes, which typically cause many such injuries. Compound fractures may be able to be managed by fixation. Open fractures are typically considered lethal unless they are treated because the open limb is exposed to infection (Rajpura et al. 2010). Under ideal circumstances, treatment is surgical fixation followed by a recovery period. For a large group of victims whose limbs are severely affected, amputation above the injury is most likely to 
save lives. It is clearly more efficient in a disaster setting than surgical fixation, and would undoubtedly cost less. However, if local hospitals have the personnel and facilities, it may be possible to do either procedure, presenting the opportunity to study survival, effectiveness, time to recovery, and cost-effectiveness. Arguably, it would be unethical to randomise patients into a surgical fixation group or an amputation group. This is because people have strong preferences about the value they place on continued life versus living with a disability. Some would clearly choose amputation with a better chance of survival, whereas others would prefer to take their chances with surgical fixation in order to save the limb. If they are able to consent, they might be given a choice of treatment, but could still be studied in comparison groups. This would have the usual consequence of potential bias in studies comparing two groups that are not randomised. However, if there were enough victims of injury who are truly indifferent regarding the two procedures and their different probable outcomes, and are willing to consent to be randomised, then the optimal research design could be used. In addition to the usual problems surrounding informed consent in a disaster, these victims may lack the capacity to consent. For those individuals, a decision still has to be made about which of the available treatments to undertake. Their preference would be unknown, so presumably the intervention more likely to save lives would be the one physicians should choose. Those patients could still be used as a comparison group for those who have capacity and make their own choice.

One of the most ethically problematic and emotional wrenching features of a medical response to a disaster is the need for triage. Victims whose condition is so serious that survival is highly unlikely are triaged out of a medical treatment group. Those who have both open fractures and a crush injury are likely to be triaged out; yet data may be gathered regarding their condition as part of the emergency response: time to death, and other information that may be useful in determining the appropriateness of triage for similar victims in the future. The prospect of gathering such information without obtaining consent appears to violate the most basic principle of research ethics. However, if observations and recording such data is considered part of a public health emergency preparedness and response, it would be perfectly acceptable. A variety of interventions may be necessary for victims of crush injuries, depending on what facilities are available. Hypotension, renal failure, metabolic abnormalities, and secondary complications are some of the medical consequences that can be managed in a hospital setting (Centers for Disease Control and Prevention 2011).

Given the large numbers of victims of an earthquake who could be studied in comparison groups where the best management is uncertain or disputed, thereby satisfying the requirement of clinical equipoise, research could yield valuable information for similar circumstances in the future. That a hospital setting can be available even when an earthquake strikes in a resource-poor country is demonstrated by the field hospital set up by the Israel Defense Forces Medical Corps in Haiti within 89 hours of the earthquake in January 2010 (Kreiss et al. 2010). It remains true, however, that if one set of best practices is implemented and studied, it could be categorised as an emergency response, part of typical public health practice; but if patients are randomised into comparison groups, it immediately qualifies as research, with all 
the procedural safeguards and protections that entails-especially the requirement of informed consent. If ever there were a situation in which research appears to be ethically permissible without obtaining informed consent, it is in a disaster where clinical equipoise exists and the victims are incapable of giving their informed consent to be studied.

\subsection{Vulnerability in Disaster Settings}

The single best justification for conducting research in the midst or in the wake of a disaster is capture by the following statement: 'To ensure effective and equitable responses to future disasters, we need to study what works and what doesn't work in present disasters' (Public Health Ethics in Disasters 2011). However, without devoting attention to the ethical concerns posed by the conduct of research in disasters, this justification is too simplistic. The first step is to address the threshold question: 1) Are victims of a disaster rendered too vulnerable by the situation to permit their inclusion in research (O’Mathúna 2010)? One reason this question is hard to answer is that there is no clear, universally accepted criterion for applying the concept of vulnerability. Let's take a brief look at what several international ethical guidance documents say about vulnerable subjects of research.

The Declaration of Helsinki is probably the best known document regarding ethics in research with human beings. However, its description of vulnerable subjects is both narrow and brief: 'Some research populations are particularly vulnerable and need special protection. These include those who cannot give or refuse consent for themselves and those who may be vulnerable to coercion or undue influence' (World Medical Association 2008 Para. 9). These characteristics of vulnerability appear more applicable to clinical research, where patients are the research subjects, than to the majority of people caught in a disaster. In addition, the description of vulnerability is too general to provide concrete guidance. What 'special protections' are appropriate or necessary? What specific features determine the ability of people to give or refuse consent? What sort of coercion might be present?

Although little empirical evidence exists regarding the extent to which people in disaster situations are vulnerable to coercion, one article gives this limited information: '[I]n a study of trauma survivors who were asked to participate in a clinical study, $19 \%$ of subjects endorsed the statement "I felt like I couldn't say no to participating", (Rosenstein 2004, p. 376). But the author of the article goes on to note that there was no comparison group in this study, and it is not possible to know if the subjects' unwillingness to refuse participation was specifically related to their having been traumatised. Still, when research is carried out in a disaster side by side with delivery of humanitarian aid, individuals may feel pressured to participate if they hold the (false) belief that assistance is contingent on their agreement to participate in research (O’Mathúna 2010).

The Council for International Organizations of Medical Sciences (CIOMS) International Ethical Guidelines for Biomedical Research include a guideline entitled 
'Research involving vulnerable persons', which states 'Special justification is required for inviting vulnerable individuals to serve as research subjects and, if they are selected, the means of protecting their rights and welfare must be strictly applied' (CIOMS 2002, p. 64). The individuals or groups mentioned in the guideline are identified as follows: 'Vulnerable persons are those who are relatively (or absolutely) incapable of protecting their own interests. More formally, they may have insufficient power, intelligence, education, resources, strength, or other needed attributes to protect their own interests' (p. 64). The chief characteristic of vulnerability this guideline identifies is a limited capacity or freedom to consent or to decline to participate in research. The criterion of 'limited freedom to consent' does not seem particularly relevant to individuals in a disaster, but some individuals may have limited capacity because of trauma, stress, or injury. The commentary following the CIOMS guideline lists numerous specific groups that may be considered vulnerable in the context of research. These include subordinate members of hierarchical groups, such as the military or students; elderly persons with dementia and residents of nursing homes; people receiving welfare benefits or social assistance, other poor people, and the unemployed; patients in emergency rooms; some ethnic and racial minority groups; homeless persons, nomads, refugees or displaced persons; prisoners; patients with incurable disease; individuals who are politically powerless; and members of communities unfamiliar with modern medical concepts. The CIOMS guideline makes no mention of victims of disasters. Members of some of the abovementioned groups may be caught in a disaster, such as elderly residents of nursing homes when Hurricane Katrina struck in New Orleans in 2005 (Khanna et al. 2005), and some people in rural areas of Japan affected by the earthquake and tsunami in March 2011. However, many people who do not fit into any of the above categories also lose their lives or are seriously injured in disasters.

Still another list of vulnerable populations appears in an ethical guidance document specific to HIV biomedical prevention research: 'Examples of populations that may have an increased vulnerability include women, children and adolescents, men who have sex with men, injecting drug users, sex workers, transgender persons, indigenous populations, the poor, the homeless, and communities from resource-poor settings in high-income and low- and middle-income countries' (UNAIDS/WHO 2007, p. 31). In this document, like the others, there is no mention of people who are caught in a disaster situation.

These various accounts of vulnerable research subjects have prompted some scepticism and a bit of a backlash regarding overuse of the concept of vulnerability. The scepticism is whether all of these various groups really should be considered vulnerable. If so, for what reason and what are the implications for involving them in research? The backlash questions whether the concept of vulnerability actually makes sense when there are so many candidates, thus prompting the question, 'Who is not vulnerable?' According to one article, 'So many groups are now considered to be vulnerable in the context of research, particularly international research, that the concept has lost force' (Levine et al. 2004, p. 44). The authors add: '... so many categories of people are now considered vulnerable that virtually all potential human subjects are included' (Levine et al. 2004, p. 46). 
Among the prominent ethical concerns about conducting research during or after disasters are those related to stress, trauma, and the ability of victims to provide informed consent (Rosenstein 2004; O’Mathúna 2010). One author concludes: 'There are no compelling data to suggest that experiencing a severe trauma, in and of itself, renders all or even most exposed individuals incapable of making autonomous decisions' (Rosenstein 2004, p. 373). If there is difficulty ascertaining individuals' capacity to provide informed consent to participate in disaster research, there is little reason to believe the problem is worse than what exists in the clinical setting. For example, the presumption in research involving psychiatric patients is that they are capable of consenting to research; evidence to the contrary must be presented if such patients are to be excluded (Appelbaum and Roth 1982). Some studies involving psychiatric patients or those who appear demented require an evaluation of individuals' capacity before they may be enrolled in research. The same procedure could be adopted in cases where doubt exists about the capacity of potential subjects in disaster research. From a practical point of view, however, this procedure would be difficult to implement when researchers seek to interview people in the chaos and confusion that typically accompany a disaster.

A different but related concern is whether individuals involved in extremely traumatic situations can anticipate the distress or discomfort they may experience as participants in disaster research (Collogan et al. 2004). Here again, the analogy with clinical and behavioural research is apt. Patients who have never undergone an invasive medical procedure as part of their medical care may nevertheless be invited to participate in a research study related to their medical condition. The procedure may cause a degree of pain or discomfort they could not foresee merely by reading or hearing the description provided in the informed consent process or document. Participants in sensitive social science research on sexuality and reproductive health or intimate partner violence are typically informed that some questions are sensitive and may make them feel uncomfortable. Yet the experience of being asked and answering such questions may provoke feelings the potential subject did not anticipate. Collogan and colleagues make a sharp distinction between trauma caused by the disaster itself, and the feelings participants in research may have when those events are recalled: 'Research participation may upset subjects but it does not traumatize them as a disastrous event would. Trauma-inducing events involve unpredictable and uncontrollable experiences, while disaster-focused research should be both predictable and highly controlled' (Collogan et al. 2004, p. 367).

The bioethics literature includes two articles that make useful distinctions when considering research involving disaster victims. The first distinction is that between 'intrinsic' and 'extrinsic' vulnerability. Intrinsic vulnerability refers to factors such as increased age, extreme youth, decreased cognitive ability, or psychosis. Extrinsic vulnerability refers to situations such as hospitalisation, imprisonment, or financial capacity (Dean and McClement 2002). According to this distinction, the large majority, if not all of the people caught in the wake of a disaster share the characteristic of 'extrinsic vulnerability.' Among that group, the elderly, infants and young children, people with physical, mental, or emotional disabilities are also 'intrinsically vulnerable.' This may be useful in determining which individuals or groups it is 
more or less ethically acceptable to involve in research during or after a disaster. The appropriateness also depends on the type of research, an additional factor noted below.

A second useful distinction in considering research with vulnerable populations introduces the idea of 'layers of vulnerability' (Luna 2009). According to Luna:

This concept of vulnerability is a relational one. That is, it concerns the relation between the person or a group of persons and the circumstances or the context. It is closely related to the situation under analysis. It is not a category or a label we can just put on ... If this is so, vulnerability should not be understood as a permanent and categorical condition, a label that is attached to someone given certain conditions (such as lack of power or incapability) that persists throughout its existence... The proposal based on layers has a related advantage. It can help in making a refined analysis of research situations. (Luna 2009, p. 129)

How would the 'layers' approach apply to the vulnerability of people caught in a disaster? The answer depends on the condition of the individuals and the nature of the research. The condition of individuals is likely to vary with the type and severity of the disaster and their exposure to harmful effects. People who are physically injured in an earthquake, for example, may be traumatised or in pain. First responders, such as fire-fighters, may be overcome by smoke or, as in the case of the terrorist attack in New York on September 11, 2001, exposed to huge quantities of dust from the collapsing buildings. People who receive very large doses of radiation may suffer immediate ill effects, as in the Chernobyl and Fukushima Daiichi nuclear plant disasters. Individuals who fall ill in an infectious disease outbreak, such as the SARS and H1N1 influenza epidemics may be too sick to answer questions or consent to provide blood samples. The characteristic of 'extrinsic vulnerability' applies to all these groups. An additional 'layer' of vulnerability would result from 'intrinsic' factors such as infirmity, disability, poor nutritional status, or an underlying disease.

The nature of the research also varies considerably. Much research conducted during or soon after a disaster is social science research: surveys, questionnaires, or in-depth interviews. This type of investigation is usually categorised as 'minimal risk' research, which is defined as involving risks of a scale ordinarily encountered in daily life or during the performance of routine physical or psychological examinations or tests' (US Department of Health and Human Services 2009 46.102(h)(i)). The harms likely to befall individuals who are asked to participate are emotional stress and invasions of their privacy by asking intrusive questions. Another type of research likely to occur in disasters such as disease outbreaks and radiation exposure involves drawing blood, also normally considered minimal risk research. Where the risks to research participants are minimal, concerns about vulnerability are a function of the ability of the subjects to consent, and not to their exposure to risks of a potentially harmful medication. On the other hand, investigations of new drugs or experimental use of existing medications to study antidotes in people exposed to attacks of anthrax or other biological agents would constitute more than minimal risk because of known side effects or unknown harmful effects. This last situation best demonstrates the 'layers' approach to vulnerability: disaster victims may be exposed to toxic or even lethal substances, they may be sick or debilitated from that exposure, and the research intervention itself carries some risks of harm and unanticipated adverse events. 
Past studies have revealed several characteristics of participants and types of studies in which disaster research has increased the subjects' potential for experiencing harms. 'These characteristics include pre-existing distress or mental illness, age (both young and old), history of multiple trauma exposures, social vulnerability, and physical injury. Furthermore, evidence suggests that repetitive research involving the same participants carries a potential for risk' (Collogan et al. 2004, p. 367). Many investigators would like to study people who have direct exposure to a particular disaster. Such individuals may be overburdened with requests to be interviewed, thereby confronting painful memories and feelings repeatedly. An enhanced potential for experiencing harms can translate into greater vulnerability, again illustrating the value of the 'layers' approach. One or more of the above characteristics of participants or types of studies can be considered an additional 'layer.' With this concept, the notion of vulnerability loses much of its vagueness and becomes more meaningful for determining what steps might be needed to protect the rights and welfare of participants in disaster research.

I contend that even where a credible case can be made that potential subjects of research are vulnerable — by using the 'layers' approach — this should not be a barrier to conducting the research. The situation is no different, in principle, from much biomedical or social science research in more usual circumstances. Medical research is conducted on severely ill patients, including those with fatal illnesses such as end-stage cancer, degenerative neuromuscular conditions, and Alzheimer's disease. When cancer patients become subjects in research they are exposed to toxic chemotherapy, drugs with significant side effects, and radiation treatment. Social science research is carried out on victims of intimate partner violence, women who have been raped, and soldiers returning from embattled war zones with post-traumatic stress disorder (PTSD). Unless all these types of research are unethical and should not be conducted because participants have layers of vulnerability, doing research in situations of disaster should not be considered unique based on the vulnerability of the subject population. As already noted, 'we need to study what works and what doesn't work' in order to prepare properly for future disasters. The authors of one article add: 'Although the risks and benefits of participation in disaster-focused research are not fully understood, most would agree that there is a significant need for additional research in the aftermath of disaster' (Collogan et al. 2004, p. 364).

The ethical guidelines for research mentioned earlier raise the question whether inhabitants of resource-poor countries are particularly vulnerable, due to poverty, their lack of accessibility to routine health care, or the poor infrastructure in the country. Do natural disasters or disease outbreaks in developing countries or poor areas in industrialised countries render the inhabitants even more vulnerable, since what they need is aid, not research (O’Mathúna 2010)? It is unquestionably true that the 2010 earthquake in Haiti, Hurricane Katrina in 2005 in New Orleans, and the 2008 earthquake in China did more damage and resulted in more loss of lives than similar events in wealthier countries or communities and in places with a far better infrastructure. Individuals in resource-poor places lack money and often basic means of subsistence, and poor countries and communities take much longer to recover 
and begin providing necessary services to the inhabitants. Although barriers to resuming electricity, food and water supplies, and medical care were also formidable in the wake of the earthquake and tsunami in Japan, they are that much worse in resource-poor countries and communities. It is unquestionably true everywhere that the inhabitants and governments in places where earthquakes, hurricanes, monsoons, and tsunamis occur require huge amounts of governmental and humanitarian assistance from the outside. But this is not an either-or situation. The need for financial, medical, and other forms of aid should not be in competition with the conduct of research. The sources of research funding may be different, and the doctors, nurses, epidemiologists, and social scientists who carry out research are often not the same people as the providers of direct care.

\subsection{Distinguishing Disaster Research from Public Health Practice}

Not all research conducted during and after disasters involves human participants. Water and soil are often tested for contaminants and radioactive substances. Investigations of sources of infectious disease outbreaks take place in the environment or in buildings. Research goes on in laboratories far from the human beings affected. One circumstance that does involve human beings is problematic, however. That is, how to distinguish public health practice in response to disasters from research in the identical situation. Both activities require gathering data from people caught in the disaster. Both activities might involve drawing blood for detection of infection or radioactive material; and both activities could involve administering an antidote or medication and studying its effectiveness. A response to disasters considered to be public health practice is typically done under governmental authority, but can be conducted by humanitarian organisations such as Médecins Sans Frontières (MSF) and the International Red Cross. The purpose of the response is to document the existence and magnitude of a public health problem in a community or region and implement appropriate measures to deal with the problem.

The distinction between public health practice and public health research is not at all clear. It remains a "grey area" because of a conceptual problem in attempting to define 'research', and because of different criteria used by different groups to determine when a public health intervention is practice and when it is research (Calain 2009; Fairchild 2003). It is important, however, for practical and ethical reasons, to make the distinction. If an activity is research, an internationally accepted requirement is that it be submitted for prior review by a duly constituted, independent research ethics committee. In addition, a strong presumption exists that voluntary, informed consent be obtained from individuals who are invited to participate in research. In contrast, when a ministry of health or other branch of government at any level responds to a disaster, there is no need for prior review by a committee and no formal consent process when individuals are surveyed or interviewed. If blood drawing or medical treatment is involved, of course individuals must agree to 
cooperate, but there are no forms to read or sign and typically no detailed procedure to disclose risks, benefits, and alternatives. The uncertainty surrounding the distinction between public health practice and research can confuse researchers as well as people involved in a disaster who are approached by public health practitioners, physicians, social workers, social scientists, or those providing humanitarian assistance.

A widely accepted definition of 'research' is the following: 'Research means a systematic investigation, including research development, testing and evaluation, designed to develop or contribute to generalisable knowledge' (US Department of Health and Human Services 2009). A key element in this definition is 'designed to contribute to knowledge that is generalisable.' The Centers for Disease Control and Prevention (CDC) in the United States uses a variation on this criterion for determining when an activity is research, referring to 'the primary intent' of the activity: 'If the primary intent is to prevent or control disease or injury or to improve a public health program, and no research is intended at the present time, the project is non-research' (Centers for Disease Control and Prevention 1999). One problem that arises for the ability to distinguish between public health response to emergencies and public health research is that what is learned in the course of the investigation may lead to generalisable knowledge, even if that was not the initial intent of the activity. A second problem is that the intent of an investigation is rarely specified in advance. As one article notes: '[T] be credible, intents need to be specified in advance of potentially harmful activities, especially when very similar or identical activities are underpinned by different intents. This would imply the existence of independent bodies or agencies capable to record expressed intents ahead of the implementation of activities, a rather unrealistic proposal when applied to fast evolving emergency settings' (Calain 2009, p. 10).

Still another ethical problem arises when an emergency response contains elements of research in addition to the primary intent - to deal with the disaster situation. It may be an urgent response to the emergency with the usual public health purpose, and at the same time be an opportunity to conduct research that could lead to knowledge applicable to future similar situations. An example could be a questionnaire accompanied by drawing blood from people who become sick and those who remain well during a disease outbreak. At the same time that public health authorities take steps to slow the spread of the disease and protect uninfected people from becoming infected, they may seek to determine whether any biological, genetic, or life style factors caused some people to get sick while others did not. The effort to make such a determination qualifies as an intention to contribute to generalisable knowledge, and therefore as research. This situation is common when biological samples are stored for dual use during a disease outbreak, resulting in a mix of research and nonresearch (Calain 2009). According to regulations governing research in the United States, if these samples are anonymised at the time they are collected, the need for ethics committee review can be waived. However, this could not readily be done in the case of outbreaks such as Marburg and Ebola, as there is a need to match separate databases accurately, along with an obligation to communicate results to concerned individuals (Calain 2009). 
In most cases in which there is an intent to conduct research, it requires preparation of a detailed research protocol. This would have to be submitted to the relevant research ethics committee for review and clearance, and the committee might request revision and resubmission of the protocol. This would delay the initiation of the response to the emergency, possibly resulting in greater harm to the population. In addition, conducting the activity as research would likely require obtaining informed consent from individuals for an activity as simple as a survey or short interview. This additional activity could take time and resources away from dealing directly with the emergency situation. If blood samples are sought from individuals, for example, to determine levels of radioactive substances, it is interesting to consider whether greater pressure could be exerted on them to allow their blood to be taken when the intervention is considered an emergency response than when it is clearly a research manoeuvre. In the latter case, the ethical requirement of the right of individuals to refuse to participate and not to be pressured or subjected to 'undue influence' should govern.

Regardless of whether a clear distinction can be made between gathering information for emergency response and gathering data for research, research ethics committees could establish a policy for disease outbreak investigations (Macklin and Cowan 2009). One possible element of the policy might be that investigators need not submit a full, detailed protocol to the committee at the outset of the study. The question of which research ethics committee(s) should be involved in approving such studies is a separate but related complication. The institution or organisation the researchers are from will almost always require ethical review, and international guidance documents typically require clearance by a local or national committee (CIOMS 2002). One or another committee could hold up the process, further delaying implementation of the research. A short statement of purpose and procedures of the investigation can be prepared and submitted for expedited review by a committee chair or other designated member. The CIOMS International Ethical Guidelines for Epidemiological Studies addresses this situation with the following observation: 'The emerging best practice for research conducted during emergency-such as population studies of outbreaks of disease or of disasters (and relief efforts) - is to establish the basic research design for various categories of research prior to the emergency. Among other benefits, this permits prior ethical review of at least the major features of the research design' (CIOMS 2009, p. 31). Even if an emergency response includes elements that unquestionably appear to be research, a duly constituted ethics oversight body could decide to waive the requirement for signed consent forms in favour of oral consent or even no consent from participants for interviews or surveys. This waiver could only be granted when the study is anonymous, that is, when individual participants cannot be identified and, as already noted, their consent must be obtained for collection of blood samples or other biological specimens.

Preparing in advance for conducting research during disease outbreaks or epidemics is not only possible, but appears to be easier than preparing in advance for research in other types of disaster. The enormous scale of destruction in earthquakes, tsunamis, and hurricanes may preclude a systematic study of the population involved. The unforeseen occurrence and suddenness of these natural events, as well 
as industrial disasters like the nuclear accident at Chernobyl and leakage of toxic chemicals at the Union Carbide pesticide plant in Bhopal, India, make it impossible to design a protocol for pre-approval by a research ethics committee. Nevertheless, a general outline or template can be prepared in advance for the predictable circumstances likely to arise in many disasters, such as the incidence of anxiety, the health consequences for displaced persons, and prevalence of PTSD. In all likelihood, the information gathered by local, regional, national, and international investigatory bodies will serve a dual purpose. Investigations in disasters serve the usual public health purpose of seeking to contain the disaster and mitigate its effects. And that information can be systematically recorded and analysed, and stored in a database, to be useful in similar circumstances in the future. Moreover, an investigation of some aspect of a disaster that begins as a public health response can transform into research as the inquiry develops. The CDC address this eventuality in its guidance on research and non-research: 'If the primary intent changes to generating generalizable knowledge, then the project becomes research' (Centers for Disease Control and Prevention 1999). This statement is an acknowledgment that the line between public health practice and public health research is fuzzy and can change as a response to a disaster evolves.

\subsection{Conclusions}

Whether research is contemplated at the outset of an emergency response to a disaster or is introduced at a later time, addressing questions related to the vulnerability of potential subjects remains an ethical requirement. One article observes that controversy exists over whether labelling a population as vulnerable may be stigmatising (Fleischman and Wood 2002). The authors correctly note that being vulnerable does not, in and of itself, raise the level of risk in a research study. However, vulnerable subjects deserve additional protections, even in research that would be categorised as 'minimal risk,' such as questionnaires or interviews. Ethical guidelines for research typically state the need for additional safeguards in the case of vulnerable subjects, but rarely specify what such protections should be. Different disasters may call for different safeguards for victims, depending on the proximity in time to the disaster in which the research takes place, the severity of injury or trauma, and other factors. When obtaining informed consent from victims in the aftermath of a disaster such as a terrorist attack, researchers should tell the subjects that the questions may be upsetting (Fleischman and Wood 2002). Similarly, researchers should plan in advance for serious emotional responses and have explicit mechanisms in place for referral to mental health professionals (Fleischman and Wood 2002). While this mechanism would almost certainly not be available for research carried out in places where there is widespread, severe damage and mental health professionals are busy attending to victims, it is feasible when survivors or relatives of people killed in the disaster are interviewed in the aftermath. In situations where there is time for a research ethics committee to review a proposal for a study, the committee could add procedural 
safeguards such as involvement of family members in the informed consent process and independent consent or research monitors (Fleischman and Wood 2002).

The types of safeguards needed for vulnerable subjects in research conducted during or after a disaster will depend on contextual factors that cannot be specified in advance. The "layers" approach to vulnerability can provide some guidance into additional protections for the rights and welfare of subjects that may be ethically necessary. One cardinal ethical principle that should always be adhered to is that research should never interfere with or delay medical care or other aid being provided to treat or prevent further harm to disaster victims.

\section{References}

Appelbaum, Paul S, and Loren H. Roth. 1982. Competency to consent to research: a psychiatric overview. Archives of General Psychiatry 39: 951-58.

Calain, Philippe. 2009. Research ethics and international epidemic response: the case of Ebola and Marburg hemorrhagic fevers. Public Health Ethics 2 (1): 7-29.

Centers for Disease Control and Prevention. 1999. Guidelines for defining public health research and public health non-research.1999. http://www.cdc.gov/od/science/integrity/docs/definingpublic-health-research-non-research-1999.pdf. Accessed 3 May 2013.

Centers for Disease Control and Prevention. 2011. After an earthquake: management of crush injuries and crush syndrome. http://wwwnc.cdc.gov/travel/content/news-announcements/reliefworkers-haiti.aspx. Accessed 9 May 2011.

CIOMS (Council for International Organizations of Medical Sciences). 2002. International ethical guidelines for biomedical research involving human subjects. http://cioms.ch/images/stories/ CIOMS/guidelines/guidelines_nov_2002_blurb.htm. Accessed 4 May 2013.

CIOMS (Council for International Organizations of Medical Sciences). 2009. International ethical guidelines on epidemiological studies. 2009. Geneva, Switzerland: CIOMS.

Dean, Ruth A., and Susan E. McClement. 2002. Palliative care research: Methodological and ethical challenges. International Journal of Palliative Nursing 8 (8): 376-380.

Fairchild, Amy. 2003. Dealing with Humpty Dumpty: Research, practice, and the ethics of public health. Journal of Law, Medicine, and Ethics 31 (4): 615-623.

Fleischman, Alan R., and Emily B. Wood. 2002. Ethical issues in research involving victims of terror. Journal of Urban Health 79 (3): 315-321.

Fleischman, Alan R. 2004. Ethical issues pertaining to research in the aftermath of disaster. Journal of Traumatic Stress 17 (5): 363-372.

Khanna, Roma, Lise Olsen, and Anita Hassan. 2005. Storm evacuations: Elderly were left with weak safety net. Houston Chronicle, December 10.

Kreiss, Yitshak., Ofer Merin, Kobi Peleg, Gad Levy, Shlomo Vinker, Ram Sagi, Avi Abargel, Carmi Bartal, Guy Lin, Ariel Bar, Elhanan Bar-On, Mitchell J. Schwaber, and Nachman Ash. 2010. Early disaster response in Haiti: the Israeli field hospital experience. Annals of Internal Medicine 153 (1): 45-48.

Levine, Carole, Ruth Faden, Christine Grady, Dale Hammerschmidt, Lisa Eckenwiler, and Jeremy Sugarman. 2004. The limitations of "vulnerability" as a protection for human research participants. The American Journal of Bioethics 4 (3): 44-49.

Luna, Florencia. 2009. Elucidating the concept of vulnerability: layers not labels. International Journal of Feminist Approaches to Bioethics 2 (1): 121-139.

Macklin, Ruth, and Ethan Cowan. 2009. Conducting research in disease outbreaks. PLoS Neglected Tropical Diseases 3 (4): e335. 
O’Mathúna, Dónal P. 2010. Conducting research in the aftermath of disasters: ethical considerations. Journal of Evidence Based Medicine 3 (2):65-75.

Pandey, Badri N., Amit Kumar, Prabha Tiwari, and Kaushala P. Mishra. 2010. Radiobiological basis in management of accidental radiation exposure. International Journal of Radiation Biology 86 (8): 613-35.

Public Health Ethics in Disasters. 2011. UNC Gillings school of global public health. Website accessed 9 May 2013 (no longer available).

Rajpura, Asim., Ihab Boutros, Tahir Khan, and Sohail A. Khan. 2010. Pakistan earthquake: experiences of a multidisciplinary surgical team. Prehospital and Disaster Medicine 25: 361-367.

Rosenstein, Donald L. 2004. Decision-making capacity and disaster research. Journal of Traumatic Stress 17 (5): 373-381.

UNAIDS/WHO. 2007. Ethical considerations in biomedical HIV prevention trials. http://www. unaids.org/en/media/unaids/contentassets/documents/unaidspublication/2012/jc1399_ethical_ considerations_en.pdf. Accessed 4 May 2013.

US Department of Health and Human Services. 2009. Code of federal regulations: Protection of human subject. http://www.hhs.gov/ohrp/humansubjects/guidance/45cfr46.html. Accessed 4 May 2013.

World Health Organization. Gender and Health in Disasters. 2002. http://www.who.int/ gender/other_health/en/genderdisasters.pdf. Accessed 3 May 2013.

World Medical Association. 2008. Declaration of Helsinki. http://www.wma.net/en/30publications/ 10policies/b3/. Accessed 4 May 2013. 